\title{
Téoros
}

Revue de recherche en tourisme

\section{Le Centre mondial d'excellence des destinations (CED)}

\section{André Vallerand et François Bédard}

Volume 26, numéro 1, printemps 2007

URI : https://id.erudit.org/iderudit/1070999ar

DOI : https://doi.org/10.7202/1070999ar

Aller au sommaire du numéro

Éditeur(s)

Université du Québec à Montréal

ISSN

0712-8657 (imprimé)

1923-2705 (numérique)

Découvrir la revue

Citer cette note

Vallerand, A. \& Bédard, F. (2007). Le Centre mondial d'excellence des destinations (CED). Téoros, 26(1), 71-72. https://doi.org/10.7202/1070999ar d'utilisation que vous pouvez consulter en ligne.

https://apropos.erudit.org/fr/usagers/politique-dutilisation/ 


\section{Le Centre mondial d'excellence des destinations (CED)}

\section{André Vallerand et François Bédard}

Le Centre mondial d'excellence des destinations (CED) est un organisme sans but lucratif, créé en décembre 2006 sur l'initiative de Tourisme Montréal et du Centre international de formation et de recherche en tourisme (CIFORT) de l'École des sciences de la gestion, Université du Québec à Montréal, deux membres affiliés de l'Organisation mondiale du tourisme (OMT).

Le CED a pour mission de contribuer à amener les destinations touristiques à l'échelle mondiale vers l'excellence, en développant leur compétitivité, en facilitant l'atteinte de leurs objectifs en matière de développement durable, en améliorant la qualité de l'expérience offerte aux touristes et en renforçant le caractère géographique d'une destination, à savoir son environnement, sa culture, son esthétique, son patrimoine et le bien-être de ses habitants.

Dans l'exercice de sa mission, le CED prolonge concrètement l'action du Conseil des destinations et des départements de l'OMT en apportant de la valeur ajoutée et de l'expertise aux politiques et aux pratiques innovatrices en matière de gestion des destinations touristiques. Par essence, le CED va agir comme catalyseur de partenariats et de collaborations avec des intervenants publics et privés, comme des organismes de gestion de destination (OGD), des experts et des associations de l'industrie touristique, des centres universitaires et, plus largement, la société civile.

\section{Responsabilités}

Les responsabilités du CED consistent à rassembler, à promouvoir, à diffuser et à développer l'expertise sur les destinations touristiques d'un réseau international de partenaires. Ultimement, les touristes, les habitants et les acteurs locaux vont bénéficier des services de ce centre et constitueront une base pour assurer le développement à long terme de la demande touristique et ainsi avoir un effet positif sur l'industrie touristique.

\section{Rôles}

Pour remplir efficacement sa mission, le Centre mondial d'excellence des destinations joue plusieurs rôles, qui pourront se mettre en place progressivement.

Dans un premier temps, le CED formera et animera un réseau de partenaires reconnus en matière d'expertise, de formation et de recherche, auprès de qui le CED aura un effet de levier, en contribuant à fédérer et à promouvoir leurs activités et en coordonnant certaines de leurs actions. II organisera une veille stratégique à l'échelle mondiale, à l'écoute et à l'avant-garde des tendances et des enjeux des destinations, pour comprendre leurs défis et leurs problématiques de gestion, de compétitivité et de développement durable. Il offrira un portail de l'expertise mondiale, qui recensera l'information et les savoir-faire sur le thème des destinations et mettra l'accent sur les pratiques et les outils dont pourraient bénéficier les acteurs publics et privés de la destination, y compris les intervenants de la société civile. II favorisera l'amélioration de la qualité des des-

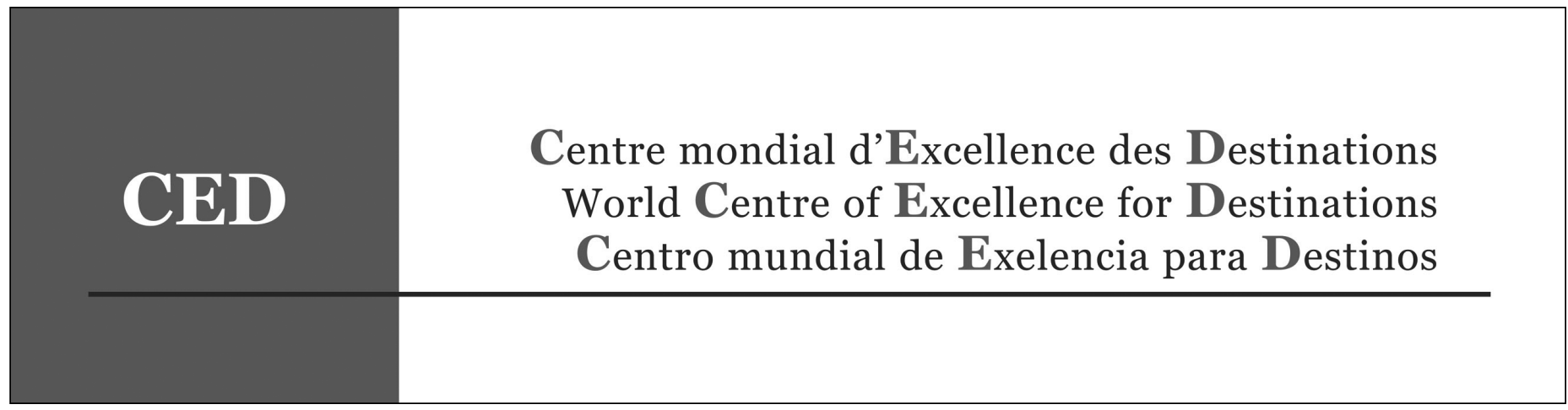


tinations, tant en ce qui concerne leurs processus que leurs pratiques de gestion. II encouragera les destinations à se faire reconnaître comme destinations d'excellence, par le biais d'un programme de certification qui sera développé sur la base de critères fiables, validés par des experts.

Dans un second temps, le CED animera un lieu d'échange et de communication entre les destinations, en initiant et en coordonnant des activités qui favorisent la communication et le partage entre les responsables et les acteurs clés des différents types de destinations touristiques à l'échelle mondiale. II assurera un leadership en matière de réflexion afin de développer l'expertise et l'innovation, en agissant comme visionnaire pour anticiper les besoins des destinations et identifier les approches novatrices, puis comme catalyseur auprès des partenaires, quand auront été identifiées des thématiques à propos desquelles il n'y a pas suffisamment d'expertise. Il promouvra la pertinence et les bénéfices des partenariats publics / privés, à l'origine de nombreux succès rencontrés par des destinations touristiques. II soulignera l'importance d'une gestion responsable, élément clé de la compétitivité et du développement durable des destinations touristiques.

\section{Réseau de partenaires}

Le CED travaillera à la mise sur pied d'un réseau de partenaires, au sein d'un grand réseau mondial, qui contribuera à enrichir le contenu des activités, des programmes et des services du CED, qui bénéficieront ainsi de son rayonnement, de son expertise de pointe ainsi que de sa capacité de promotion internationale. Ces partenaires, qui seront sélectionnés en raison de leur expertise et de leur excellence, comprendront des administrations nationales du tourisme, des experts du secteur privé, des associations de l'industrie, des OGD, des centres universitaires, tous détenteurs de l'excellence et reconnus pour leur expertise sur les destinations touristiques, et des groupes de la société civile, qui représentent la préservation du patrimoine historique, la gestion du territoire, l'architecture, l'esthétique et l'agriculture, ainsi que d'autres contributeurs à la qualité de l'expérience touristique d'une destination.

\section{Bénéficiaires du CED}

Les clientèles susceptibles de bénéficier du Centre mondial d'excellence des destinations sont les administrations nationales du tourisme, les OGD, aux paliers national, régional et local, les autorités publiques aux paliers local et régional, les entreprises, les associations et les organismes de l'industrie touristique qui œuvrent dans des catégories d'activités dont le succès est souvent lié à la performance des destinations touristiques (hébergement, organismes de loisir, expositions et congrès...), les institutions des domaines de l'enseignement, de la formation et de la recherche en tourisme, les organisations non gouvernementales (ONG), la société civile, les résidants et les touristes.

\section{Services et produits}

D'une part, les produits et services seront développés dans une optique de valeur ajoutée et de partenariat avec les détenteurs d'expertise et les institutions de recherche, qui constituent le réseau.
D'autre part, le développement des produits et services assurera la synergie et évitera la duplication avec les programmes de l'OMT.

Les produits et services envisageables dans un premier temps incluent la veille stratégique, un portail électronique, des recherches comparatives (analyses qualitatives et quantitatives), des outils de gestion ou de marketing, un programme de certification de l'excellence des destinations touristiques et des outils liés à l'intendance, pour l'industrie et les communautés.

Au fur et à mesure des possibilités d'autofinancement des activités et des possibilités émanant des partenaires et des activités de l'OMT, les produits et services suivants pourront être mis en place : recherches spécifiques et études de marché, conférences, séminaires et forums, démonstrations pratiques de succès touristiques imputables à des partenariats publics / privés, programmes de formation et publications dans plus d'une des langues officielles de l'OMT...

Le CED constitue une occasion d'enrichir et d'accroître les activités suivantes, d'ores et déjà réalisées par l'OMT, notamment l'assistance technique procurée par les départements de l'OMT, le programme de certification Sbest de la Fondation OMT.Thémis, les programmes de formation offerts par les différentes instances de l'OMT et les conférences, les séminaires, les forums et les publications de l'OMT.

Certains produits et services pourraient être proposés gratuitement aux membres de l'OMT, afin de représenter une valeur ajoutée, d'autres produits et services seraient vendus aux différentes catégories de bénéficiaires visées dans le but d'assurer au minimum l'équilibre financier du centre.

\section{Financement}

Outre les ressources humaines, matérielles et financières provenant des deux organismes fondateurs, le CED a obtenu dès le départ une contribution financière du secteur public (ministère du Tourisme du Québec, ministère des Affaires municipales et des Régions et Développement économique Canada - région Québec) et du secteur privé (Bell Canada).

\section{Structure}

Le Centre mondial d'excellence des destinations a le statut d'un organisme sans but lucratif incorporé au Québec. II est régi par des principes d'autonomie (financière, de gestion et de programmation) et de transparence, d'intégrité intellectuelle et de neutralité.

\section{Fonctionnement}

La structure opérationnelle du CED, dont le centre administratif est situé à Montréal, est légère et flexible, afin de faire jouer au maximum l'effet de levier provenant du groupe des partenaires qui composent le centre et de réunir les financements et les ressources nécessaires.

André Vallerand est président du Centre mondial d'excellence des destinations (CED).

François Bédard est professeur au département d'études urbaines et touristique de I'UQAM et directeur général du CED. 